\title{
Peliosis hepática focal: Caso clínico radiológico
}

\author{
ROBERTO MIRANDA ${ }^{1}$, JUAN STAMBUK ${ }^{2}$, \\ MARÍA TERESA VIAL ${ }^{3}$, ANDRÉS MIRANDA $^{\text {a }}$ \\ Peliosis hepatis.
Report of one case
}

Peliosis hepatis is a rare vascular condition of the liver characterized by the presence of cystic blood-filled cavities distributed randomly throughout the liver parenchyma. We report a 46 year old female, with dyspepsia. An abdominal Doppler ultrasound showed a hyper vascularized solid liver nodule of $5 \mathrm{~cm}$ diameter. Magnetic resonance imaging and CT scan showed the same hyper vascularized nodule. With the possible diagnoses of primary hepatocellular carcinoma or focal nodular hyperplasia, the patient was subjected to an excision of hepatic segment VI, where the nodule was located. The pathological diagnosis of the surgical piece was a peliosis hepatis.

(Rev Med Chile 2010; 138: 1135-1139).

Key words: Liver neoplasms; Magnetic resonance imaging; Peliosis hepatis; Ultrasonography, Doppler.

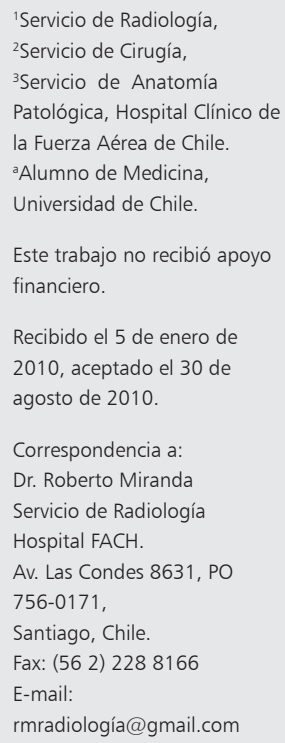

L a peliosis hepática (peliosis hepatis) es una enfermedad vascular poco frecuente, caracterizada por nódulos hepáticos únicos o múltiples, constituidos por sinusoides dilatados y múltiples espacios lacunares llenos de sangre. Las manifestaciones clínicas son variadas, frecuentemente asintomática ${ }^{1}$. Su aspecto radiológico es variable y poco específico. Puede afectar también bazo, linfonodos e incluso la médula ósea, pleura, pulmón, riñón, estómago y suprarrenales ${ }^{1}$. Se presenta un caso de peliosis hepática demostrada con histología.

\section{Caso clínico}

Mujer de 46 años con antecedentes de pólipo gástrico hiperplásico resecado hace 3 años. Portadora de otitis media crónica. Apendicectomizada en 1995. Usuaria de anticonceptivos orales por 17 años. Sin antecedentes alcohólicos ni tabáquicos. Por leves síntomas dispépticos se realizó una ultrasonografía (US) abdominal encontrándose un nódulo sólido hepático hipoecogénico de $5 \mathrm{~cm}$, hipervascular al doppler (Figura 1).

La resonancia magnética (RM) mostró un nódulo de 5 x $6 \mathrm{~cm}$ en el borde inferior del segmento VI, levemente hiperintenso y heterogéneo en T1, sin caída de señal en secuencia fuera de fase, levemente hiperintenso en T2 (Figura 2). Tras la inyección de gadolinio intravenoso en fase arterial se mostró un importante realce centrípeto con un anillo hipointenso periférico que lo delimitaba parcialmente. En fase venosa se reforzó su señal en forma homogénea y en fase de equilibrio apareció hiperintenso homogéneo (Figura 3). Se planteó la posibilidad de un hemangioma atípico por la poca hiperseñal en T2 y el anillo hipointenso periférico en fase arterial, no habitual de los hemangiomas.

Las pruebas hepáticas, hemograma, HIV, virus hepatitis $\mathrm{B}$ y C y alfa feto proteína fueron normales.

La tomografía computada (TC) sin contraste mostró un nódulo hipodenso homogéneo, sin calcificaciones. En fase arterial, era hipervascular heterogéneo con llene total con un anillo hipodenso periférico. En fase portal y tardía mostraba 


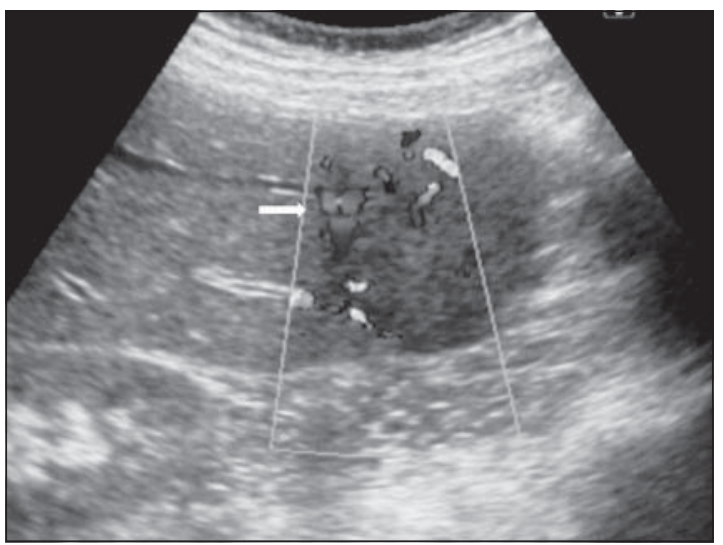

Figura 1. US con Doppler: nódulo sólido hipoecogénico vascularizado segmento 6 del hígado (flecha). lavado de contraste central con moderado refuerzo periférico, con un tenue anillo denso periférico (Figura 4). Se planteó la posibilidad de una lesión hepatocelular, hiperplasia nodular focal o hepatocarcinoma.

En discusión clínico radiológica se decidió realizar una cirugía resectiva del segmento VI, evolucionando sin incidentes.

Para el estudio anátomo- patológico se recibió un fragmento de tejido hepático de $8,5 \times 7 \times 4$ $\mathrm{cm}$. Al corte se distinguía un área nodular rosado violácea de 4,5 $\mathrm{cm}$ de eje mayor, de arquitectura levemente irregular en relación al parénquima hepático circundante. El aspecto microscópico reveló múltiples espacios irregulares con alteración de la
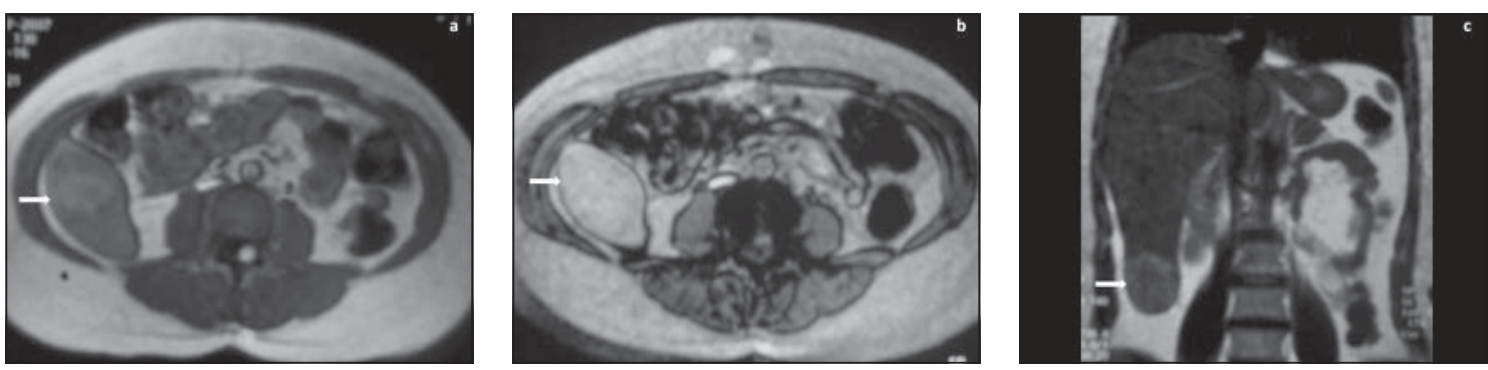

Figura 2. RM secuencias T1 dentro y fuera de fase y secuencia T2 coronal. Nódulo hepático sólido (flechas) levemente hiperintenso en T1 (a), sin contenido adiposo (b), hiperintenso en T2 (c).
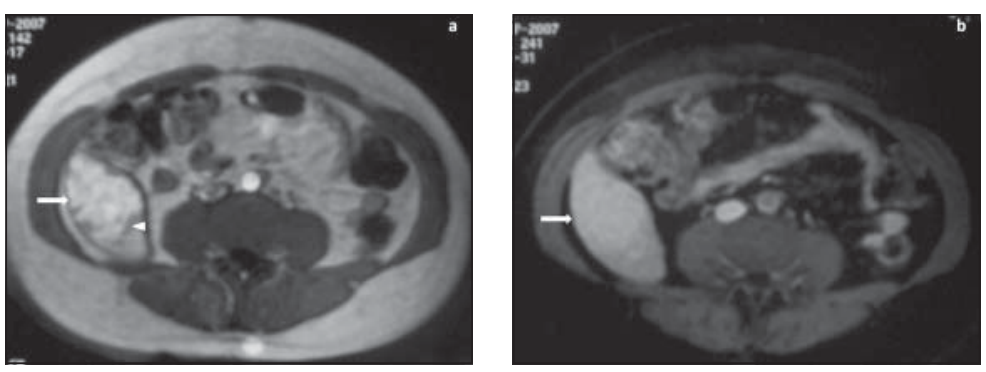

Figura 3. RM con gadolinio en fases arterial y tardía. El nódulo es hipervascular (a) con un halo hipodenso (cabeza de flecha), que muestra refuerzo homogéneo en fase tardía (b).
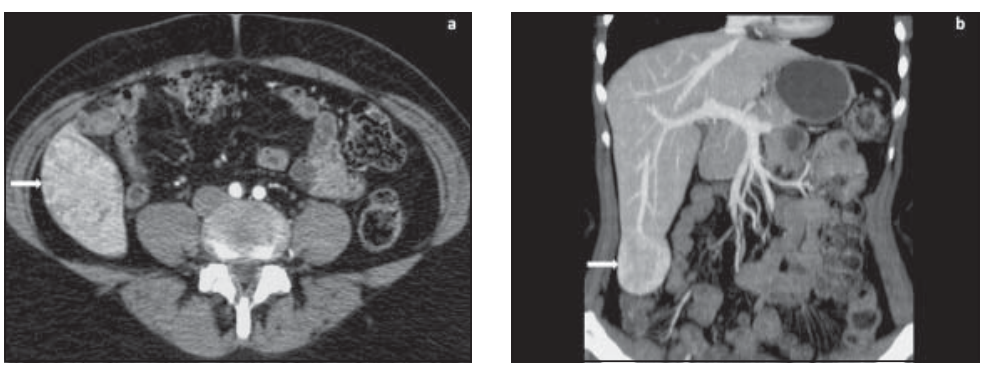

Figura 4. TC en fase arterial y venoso. Nódulo hipervascular con halo hipodenso periférico (a) y, lavado central con refuerzo periférico en fase venosa (b). 

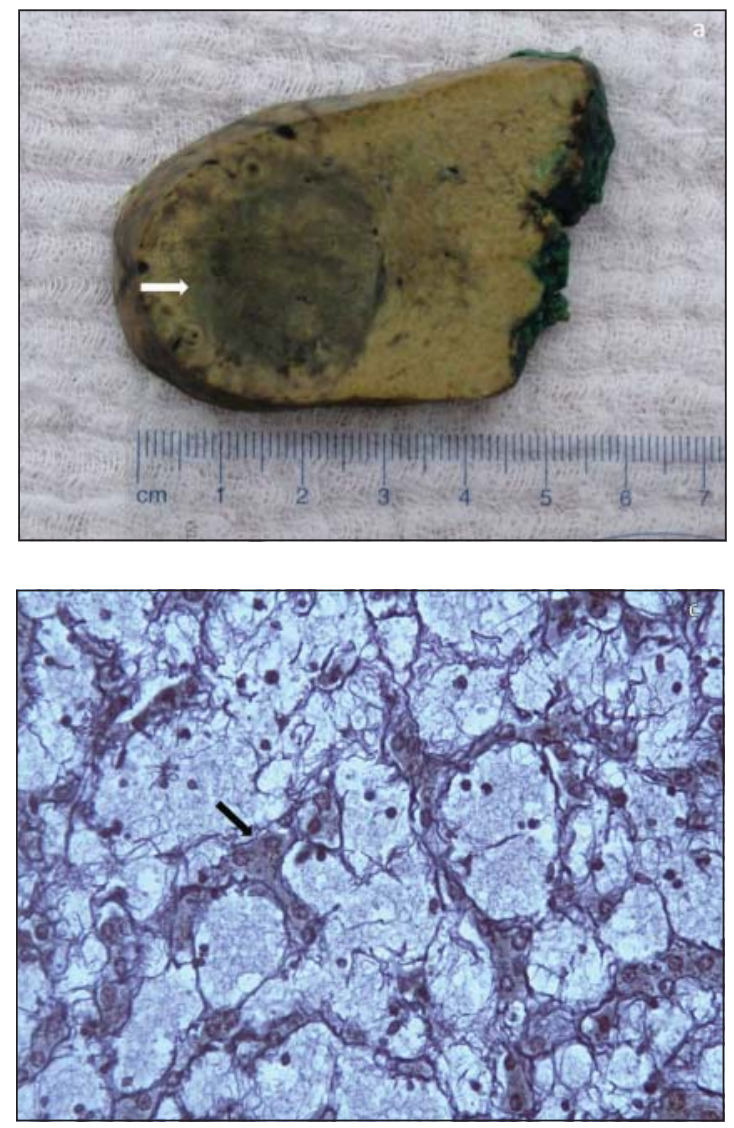

trama reticular, que contenían material hemático $\mathrm{y}$ algunos macrófagos. Estaban intercalados y en partes rodeados por tejido hepático conservado con esteatosis focal macrovacuolar, hallazgos que orientaban a una peliosis hepática (Figura 5).

Al control a los 18 meses, la paciente se encuentra asintomática. La US de control no muestra lesiones focales. Suspendió el uso de anticonceptivos.

\section{Discusión}

Un problema clínico radiológico en la práctica diaria, es el hallazgo de lesiones nodulares hepáticas que nos enfrentan a distintos diagnósticos diferenciales ${ }^{2}$. Es conocido el contexto clínico y radiológico de varias lesiones focales hepáticas de frecuencia relativamente alta, principalmente los hemangiomas, la hiperplasia nodular focal y

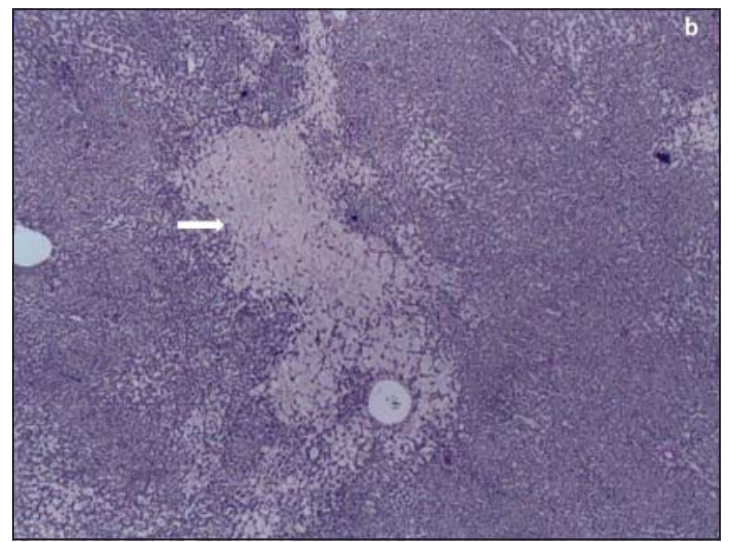

Figura 5. Anatomía patológica. Corte sagital de la pieza a nivel del segmento 6 del hígado (a) muestra un nódulo con zonas violáceas en su espesor. Preparado histológico con zonas lacunares irregulares intercaladas con tejido hepático (b) y lagunas llenas de material hemático separadas por trama reticular (c).

los hepatocarcinomas ${ }^{2}$. Sin embargo, lesiones de ocurrencia menos frecuente nos obligan a usar una batería más amplia de métodos diagnósticos, que muchas veces concluyen en la resección quirúrgica para estudio anátomo-patológico ${ }^{3}$.

Nuestra paciente, sin molestias clínicas relevantes es controlada por el antecedente de pólipo gástrico resecado 3 años antes. Hace 5 años se realizó un US abdominal, que fue normal. Usuaria habitual de anticonceptivos orales desde los 28 años. Su lesión actual es descubierta gracias a la precautoria solicitud de un US abdominal sin tener alteraciones en los exámenes de laboratorio. Es operada dado el aspecto poco específico de la lesión en 3 diferentes métodos de imagen, decisión tomada en consenso clínico radiológico.

La peliosis hepática (del Griego pelios, púrpura por su aspecto oscuro al corte) es una enfermedad vascular infrecuente, de causa desconocida, caracterizada por un compromiso multifocal del hígado, 
con la presencia de múltiples pequeños nódulos de aspecto seudoquístico. Aunque habitualmente la forma de presentación es multifocal, la peliosis focal con lesión única ha sido descrita con mayor frecuencia en el último tiempo ${ }^{3}$.

El estudio anátomo-patológico muestra dilataciones quísticas de los sinusoides llenos de glóbulos rojos, rodeados por cordones hepatocitarios que generalmente no tienen recubrimiento endotelial o bien, presentan revestimiento parcial por reendotelización. La expansión por rotura de la pared endotelial o necrosis de los hepatocitos, definen la extensión y aspecto de la lesión ${ }^{1,4,5}$.

El 20-50\% de los casos no tiene una causa conocida. Se ha visto asociación de peliosis hepática con uso de esteroides anabolizantes, corticoides, anticonceptivos orales, tamoxifeno, azatioprina, dietil-estilbestrol, 6-tioguanina, 6-mercaptopurina y metotrexato. También con tóxicos como polivinilos (PVC), arsénico y torio. Aparece en enfermedades debilitantes como tuberculosis, lepra y varias neoplasias fundamentalmente el hepatocarcinoma, pacientes con síndrome de inmunodeficiencia adquirida (SIDA), asociado a infecciones oportunistas (peliosis bacilar) causadas por Bartonella henselae y Bartonella quintana y otras como diabetes, sprue, vasculitis necrotizantes, alteraciones hematológicas o después de trasplante renal o cardíaco ${ }^{1,6}$.

Desde el punto de vista clínico, generalmente son pacientes asintomáticos y su diagnóstico es un hallazgo incidental en radiología o autopsias. No tiene predilección por sexo ni edad aunque es más frecuente en adultos ${ }^{1}$. La repercusión clínica más importante es por complicaciones dentro de las que encontramos la insuficiencia hepática por compromiso multifocal o la hemorragia por rotura espontánea ${ }^{1,5,7}$. Si se asocia a una etiología conocida, al suspenderla, las lesiones pueden regresar espontáneamente ${ }^{3}$. Una peliosis no tratada puede tener una evolución fatal ${ }^{7,8}$.

El diagnóstico por imágenes de la peliosis es variable dependiendo del estado evolutivo y si es unifocal o multifocal ${ }^{1,2,9,10}$. A la US puede ser hipoecogénica o hiperecogénica, homogénea o heterogénea si tiene focos hemorrágicos. Al doppler puede tener flujo periférico o central ${ }^{1}$.

La TC muestra focos hipodensos únicos o múltiples y su comportamiento vascular también es variable, pudiendo tener un aspecto hipovascular o hipervascular para hacerse progresivamente iso- densa en fase tardía. Puede tener un llene globular que más frecuentemente es de tipo centrífugo pero también puede ser centrípeto, similar a los hemangiomas. En fase tardía puede ser hiperdensa por ectasia venosa ${ }^{1,3}$.

La RM también tiene un aspecto variable. En $\mathrm{T} 1$ puede ser hipo o hiperintensa dependiendo si existe hemorragia. En T2 generalmente es hiperintensa. Con gadolinio en fase arterial puede aparecer también como hipointensa por hipovascularización principalmente en las multifocales o hiperintensa en las lesiones únicas hipervasculares. Hay acumulación de contraste en las fases venosas y tardías con un aspecto hiperintenso variable $e^{1-3,9,10}$.

La caracterización de una masa hepática es siempre obligatoria. En el caso de una peliosis el aspecto radiológico es variable, por lo que la biopsia está siempre indicada ${ }^{5}$. Hay bibliografía que demuestra la regresión espontánea al suprimir la causa asociada ${ }^{3}$. Con el estudio anátomo-patológico podremos llegar al diagnóstico definitivo.

El chequeo preventivo con imágenes en pacientes que reciben tratamientos farmacológicos prolongados (anticonceptivos orales en nuestra paciente), puede ayudar a reconocer entidades patológicas no siempre sospechadas.

\section{Diagnóstico diferencial}

Los distintos aspectos radiológicos de la peliosis plantean varios diagnósticos diferenciales que incluyen lesiones tumorales benignas y malignas $^{1,2,11}$. En general la peliosis no produce efecto de masa, aunque puede existir en las lesiones únicas y de mayor tamaño.

Dentro de las lesiones benignas el hemangioma es la lesión focal hepática más frecuente. Con contraste tiene un llenado nodular periférico, sin un anillo que separe la lesión vascular del resto del hígado, además del característico llene centrípeto, opuesto al de la peliosis múltiple. Además son marcadamente hiperintensos en secuencias T2 diferente de nuestro caso ${ }^{1,9}$.

En los adenomas el antecedente de uso de anticonceptivos es muy importante; las secuencias de RM que muestran la presencia de grasa microscópica ayuda a diferenciarlos 9 .

La hiperplasia nodular focal es la segunda lesión más frecuente del hígado. Son lesiones muy vascularizadas, con llene muy homogéneo en 
fase arterial, isodensas en la venosa y en la tardía, frecuentemente con una cicatriz central hiperintensa en $\mathrm{T} 2^{9}$.

Los abscesos hepáticos se presentan en un contexto clínico diferente.

Las lesiones malignas incluyen al hepatocarcinoma, en un contexto clínico diferente; las imágenes muestran un lavado rápido de contraste desde la fase arterial a la tardía donde aparecen hipodensos seudoencapsulados, con un patrón generalmente heterogéneo en $\mathrm{RM}^{2,9}$.

Las metástasis también aparecen en un contexto clínico diferente.

No se recomienda la punción percutánea de la peliosis por el riesgo hemorrágico ${ }^{5}$.

En resumen, la peliosis hepática es una patología de difícil diagnóstico, generalmente asintomática, como en nuestro caso. Simula otras lesiones nodulares hepáticas. Debe ser considerada en el diagnóstico diferencial de las lesiones nodulares hepáticas de presentación atípica. Nuestro caso podría estar asociado al uso prolongado de anticonceptivos $^{12,13}$. La resección quirúrgica nos permitió confirmar el diagnóstico y evitar complicaciones.

\section{Referencias}

1. Iannaccone R, Federle M, Brancatelli G, Matsui O, Fishman E, Narra V. Peliosis hepatis: spectrum of imaging findings. AJR Am J Roentgenol 2006; 187: w43-52.

2. Khaled ME, Yuming Y, Brown J. Focal hepatic lesions: diagnostic value of enhancement pattern approach with contrast enhanced $3 \mathrm{~d}$ gradient-echo MR imaging. Radiographics 2005; 25: 1299-320.
3. Savastano S, Velo E, Rettore C, Altavilla G. Pseudotumoral appearance of peliosis hepatis. AJR Am J Roentgenol 2005; 185: 558-60.

4. Edwards R, Colombo T, Greaves P. "Have you seen this?" Peliosis hepatis. Toxicol Pathol 2002; 30: 521-3.

5. Abbott R, Levy A, Aguilera N, Gorospe L, Thompson W. Primary vascular neoplasms of the spleen: radiologicpathologic correlation. Radiographics 2004; 24: 1137-63.

6. Dal Mo Yang, Dong Hae Jung, Chul Hi Park, Jee Eun Kim, Soo Jin Choi. Imaging findings of hepatic sinusoidal dilatation. AJR Am J Roentgenol 2004; 183: 1075-7.

7. Smathers R, Heiken JP, Lee JK. Computed tomography of fatal hepatic rupture due to peliosis hepatis. J Comput Assist Tomogr 1984; 8: 768-9.

8. Tsirigotis P, Sella T, Shapira M, Bitan M, Bloom A, Kiselgoff D, Levin M, Libster D. Peliosis hepatis following treatment with androgen-steroids in patients with bone marrow failure sindromes. Haematologica 2007; 92.

9. Siegelman E. Body MRI. United States: Editorial Saunders; 2005. p. 4-62.

10. Schneider G, Luigi G, Saini S. Resonancia Magnética del Hígado. Berlin, Alemania: Editorial Springer-Verlag; 2006. p. 17-264.

11. Brancatelli G, Baron R, Peterson M, Marsh W. Helical CT screening for hepatocellular carcinoma in patients with cirrhosis: frequency and causes of false-positive interpretation. AJR Am J Roentgenol 2003; 180: 100714.

12. Muñoz M, Rodríguez N, Tordecilla J, Ureta E, Rizzardini C, Soto V, Wensioe K. "Peliosis Hepatis" como complicación del uso de anticonceptivos orales en una paciente con mielodisplasia. Rev Chil Pediatr 2009; 80: 354-60.

13. Pérez-Holanda S, Tojo S, Calleja M, Fernández A, Fernández P, Martínez D. Peliosis Esplénica, una entidad poco frecuente. Rev Esp Enferm Dig 2007; 99: 359-67. 